\title{
ANATOMICAL AND VISUAL OUTCOME OF COMBINED PHACO- VITRECTOMY VS VITRECTOMY ALONE
}

\author{
IRFAN MUSLIM, ASAD ASLAM KHAN, NASIR CHAUDHRY \\ Department of Ophthalmology, King Edwards Medical University - Mayo Hospital, Lahore
}

\begin{abstract}
Background: Separation of retina from retinal pigment epithelium is described as retinal detachment. Various studies have evaluated the results of combined phaco-vitrectomy, the combined approach allows faster visual recovery and has cost effectivity for patient.

Objective: To compare the anatomical and visual outcome of combined phacoemulsification and vitrectomy vs vitrectomy alone

Methods: This prospective randomized controlled trial was performed at Eye Unit II Mayo Hospital Lahore from August 2019 to February 2020. 36 patients full filling the inclusion criteria were divided into two groups 18 patients each. Group A was of combined Phaco-vitrectomy and Group B was of vitrectomy only. The Mann-whitney test was applied and $\mathrm{P} \leq 0.05$ was considered statistically significant.

Results: Best corrected visual acuity at first week post operatively was significantly lower in patients in which we used gasses as internal tamponade. The BCVA at 1 month showed improvements in both groups, however the BCVA in group $\mathrm{B}$ was significantly lower than group A, P-value being $<0.05$. The BCVA at 3 month showed improvements in both groups, however the BCVA in group B was significantly lower than group A, P-value being < 0.05 . In both groups redetachment rates were comparable. The P-value was $>0.05$ and thus the difference between two groups was insignificant. Conclusion: The visual outcome of group A was significantly better than group B while the anatomical outcome was the same.
\end{abstract}

Keywords: Phacoemulsification, Three ports pars plana vitrectomy, best corrected visual acuity, retinal detachment.

How to cite this article: Muslim I, Khan AA, Chaudhry N. Anatomical and visual outcome of combined PhacoVitrectomy vs Vitrectomy alone. Pak Postgrad Med J 2020;31(4): 204-209

This is an Open Access article distributed under the terms of the Creative Commons Attribution License (http://creativecommons.org/licenses/by/3.0), which permits unrestricted use, distribution, and reproduction in any medium, provided the original work is properly cited.

DOI: http://doi.org/10.51642/ppmj.v31i04.362

Correspondence to: Irfan Muslim,

Senior Registrar, Unit II,

Department of Ophthalmology, King Edwards Medical

University - Mayo Hospital, Lahore, Pakistan.

Email:dr.irfanmuslim@gmail.com

\section{INTRODUCTION}

Separation of Neurosensory retina from RPE (Retinal Pigment Epithelium) is described as retinal detachment. Rhegmatogenous retinal detachment, which happens due to a break in the retina, is the most common type. This break in the neurosensory retina allows in the passage of fluid in space between the RPE and neurosensory retina. Recently Pars plana vitrectomy has emerged as a preferred surgical treatment for management of uncomplicated primary rhegmatogenous retinal detachment. Most important advantages are intraoperative identification and sealing of the retinal breaks. Along with improved view of the peripheral retina. ${ }^{1}$

Stereopsis, visual details of surgical field, depth of focus and visual field itself are key dependent points of pars plana vitrectomy, and thus the essential requirement for successful and complete Posterior segment surgery is clarity of the ocular media. ${ }^{2}$ There are multiple limitations to this which include preexisting cataract that may limit visualization of the retina, the normal clear crystalline lens may limit the removal of peripheral vitreous and traction relieved, and thus it may be more difficult to identify and treat peripheral pathology in phakic eyes. ${ }^{1}$ It is important to note that even if there was 
no visually significant cataract prior to vitrectomy, various studies and clinical experience has shown that these patients will ultimately develop visually significant cataract and will require cataract surgery in future. Thus, patient may have to go through multiple surgeries burden. ${ }^{3-4}$

Cataract surgery such as phacoemulsification is associated with higher risk of complications in vitrectomized eyes because of unstable AC depth and lack of vitreous support. Also, combined phacovitrectomy poses challenges such as prolonged surgical time and increased post-op inflammation. There is still debate that which is better, or either these procedures are done separately or together as both procedures have its advantages and disadvantages. ${ }^{5}$

Advances in instrumentations and small incision approach makes combined procedure easier and a widely accepted approach to combine both surgeries. ${ }^{6}$ In many cases cataract surgery is a preferred option to manipulate the peripheral retina during vitrectomy as it provides more space and avoids the risk of lens touch during pars plana vitrectomy. ${ }^{7}$ Various studies have evaluated the results of combined phaco-vitrectomy, the combined approach allows faster visual recovery and has cost effectivity for patient. ${ }^{8-9}$

We made two groups of patients with phakic Rhegmatogenous RD, in the Group A, the first Group, phaco-vitrectomy for retinal detachment in the same sitting whereas in the Group B, the second Group, only vitrectomy will be performed for retinal detachment. We compared their postoperative visual and anatomical outcomes.

The rationale of this study is to compare the anatomical and visual outcome of combined phacoemulsification and vitrectomy vs vitrectomy alone and to demonstrate which of the two is better in terms of visual and anatomical outcome.

\section{METHODS}

This prospective randomized controlled trial was performed at Eye Unit II Mayo Hospital Lahore (tertiary care eye hospital in Lahore), Pakistan from August 2019 to February 2020. Thirty-six patients full filling the inclusion criteria were selected and their consent was taken on consent proforma. No ethical issues were involved. Two groups Group A and Group B of 18 patients each were created. Patients were randomly allocated to each group. Group A was of combined Phaco-vitrectomy and Group B was of vitrectomy only. Consent for phacoemulsification with already discussed vitrectomy was taken separately. Single vitreoretinal surgeon performed all the surgeries. 3 months follow-up of all patients was done post-surgery.
Patients who presented with tractional Retinal Detachment, Exudative Retinal Detachments, dropped nucleus, penetrating injury and endophthalmitis were not included in this study. Both the Groups had clear crystalline lens.

Alcon Accurus Vitrectomy machine was used for performing both cataract and vitreo-retina surgery. Oculus BIOM 2 was used for retinal visualisation, with Oculus SDI Inverter 2. 23G transconjunctival pars plana vitrectomy was performed for all the patients with Rhegmatogenous retinal detachments.

In these surgeries other adjuncts which were used included gasses such as, SF6 (pure sulphur hexafluoride), C3F8 (pure octafluoropropane) and silicon oils such as, Silicone oil $1.000 \mathrm{cS}$, and heavy liquid (HPF10 high purity perfluorodecalin). For endolaser we used $532 \mathrm{~nm}$ green laser.

In all cases of Group, A, before starting phacoemulsification, three ports were made at $3.5 \mathrm{~mm}$ form the limbus with 23-gauge cannulas with self-sealing at standard quadrants. During phacoemulsification clear corneal incisions were given and all were $3.2 \mathrm{~mm}$ in length. Cataract surgery was performed and implantation of intraocular lens was done at the end of pars plana vitrectomy. Foldable square edged acrylic hydrophilic intraocular lenses were implanted. 10/0 Nylon suture was used to secure the wounds at the end of cortical cleanup to prevent any iris prolapse and AC leak. At the end all vicryl 6/0 suture was used to close all of the vitrectomy. VA pre-operatively and post- operatively were performed with Log-MAR VA chart.

We used SPSS statistical software for data analysis. Numerical data was represented as mean \pm standard deviation. For qualitative variables percentages and frequencies were calculated. The Mann-whitney test as test of significance was applied and $\mathrm{P} \leq 0.05$ was considered statistically significant.

\section{RESULTS}

A total of 36 eyes of 36 patients were included in this study. Descriptive statistics combined are given in the following table

AGE

\begin{tabular}{ccccc}
\hline No & Minimum & Maximum & Mean & $\begin{array}{c}\text { Std. } \\
\text { Deviation }\end{array}$ \\
\hline 36 & 42.00 & 66.00 & 54.9444 & 6.07075 \\
\hline
\end{tabular}

GENDER

\begin{tabular}{lcc}
\hline & Frequency & Percent \\
\hline Male & 20 & 55.6 \\
Female & 16 & 44.4 \\
Total & 36 & 100.0 \\
\hline
\end{tabular}


Eighteen eyes were included in both Group A and Group $B$ respectively. The mean age was $53.7 \pm 5.60$ years in Group A and 56.1 \pm 6.18 years in Group B respectively (pvalue $>0.05$ ). So, in both the groups the mean age of the patients did not differ significantly. In group A, male to female ratio was $1.25: 1$ whereas in group B male to female ratio was also 1.25:1.

BCVA done Pre-operatively for Group A and B is shown in the following Figure 1. P-value was $>0.05$ which was insignificant.

Best corrected visual acuity at first week post operatively was significantly lower in patients in which we used gasses as internal tamponade as compared those in which we used silicon oil as tamponade. This decrease in visual acuity was attributable to inherent property of intraocular gasses to impair vision. Silicon oil causes intermediate decrease in visual acuity. First week Post- operative BCVA distribution among the groups is given in the following figure

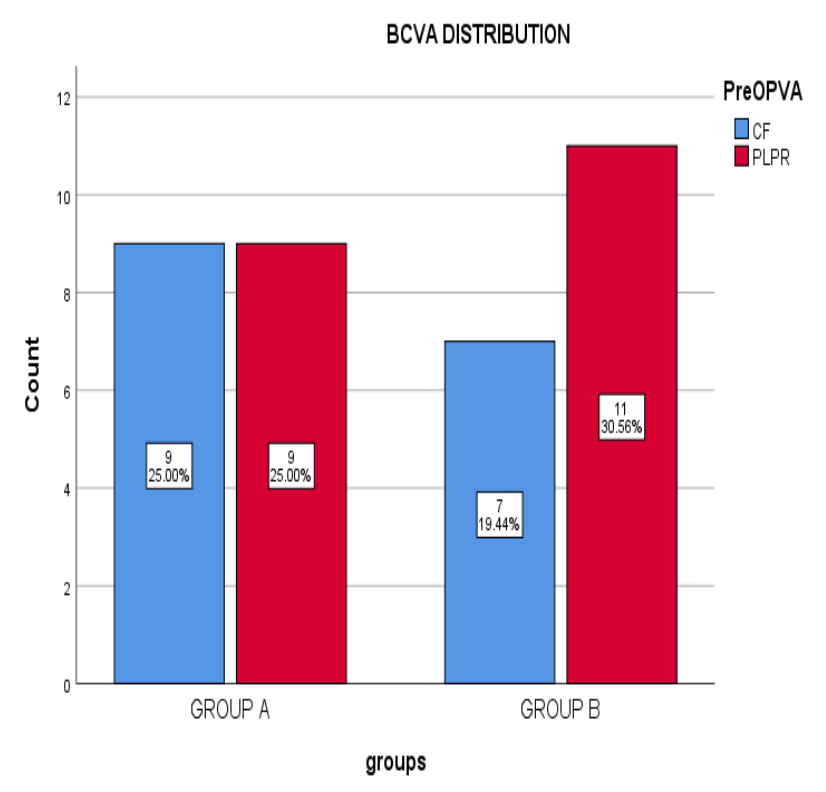

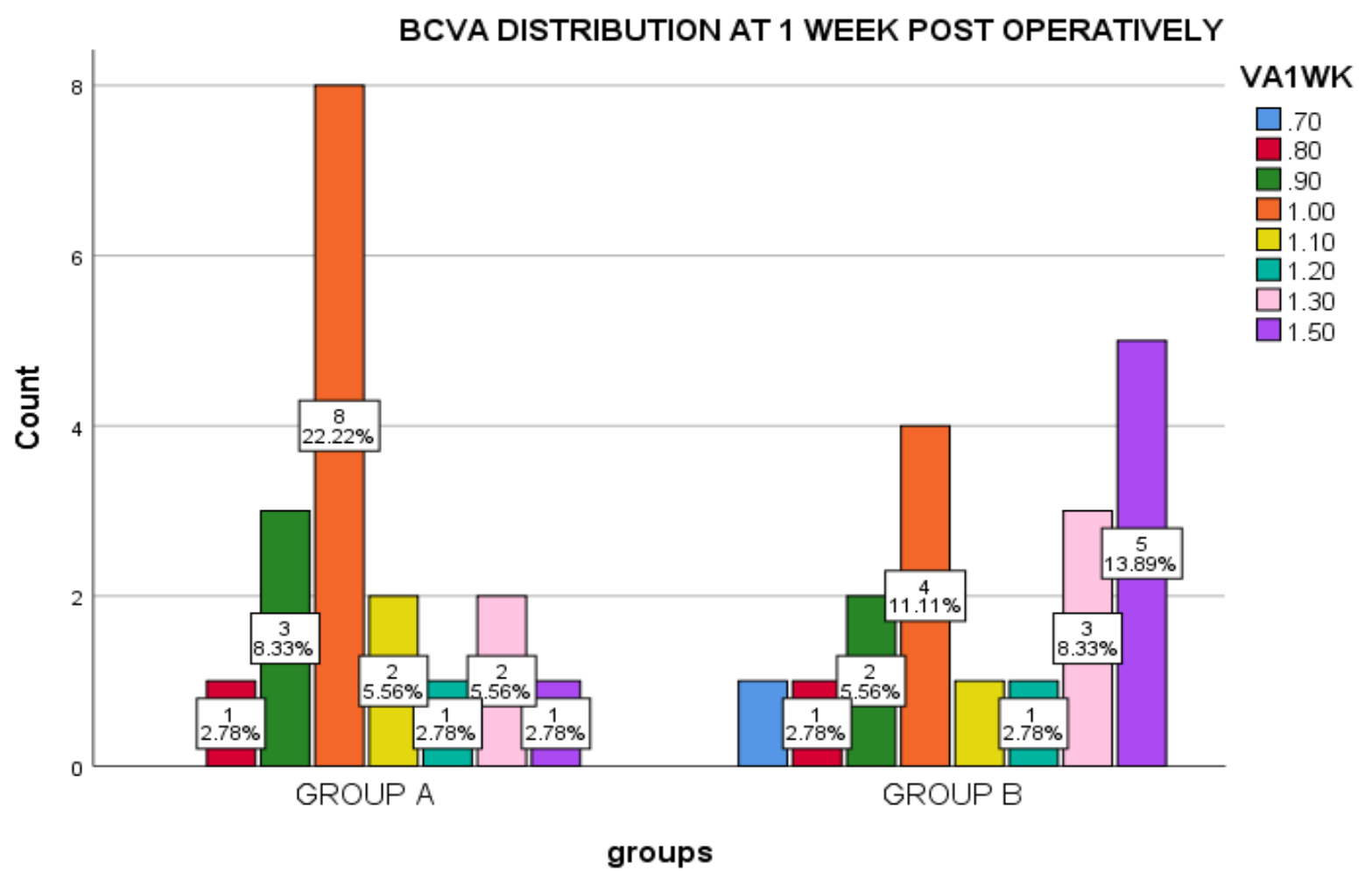

The was no statistically significant difference between the two groups as the $\mathrm{p}$ value was $>0.05$. The BCVA distribution at One month's follow-up is shown in Figure 2. 


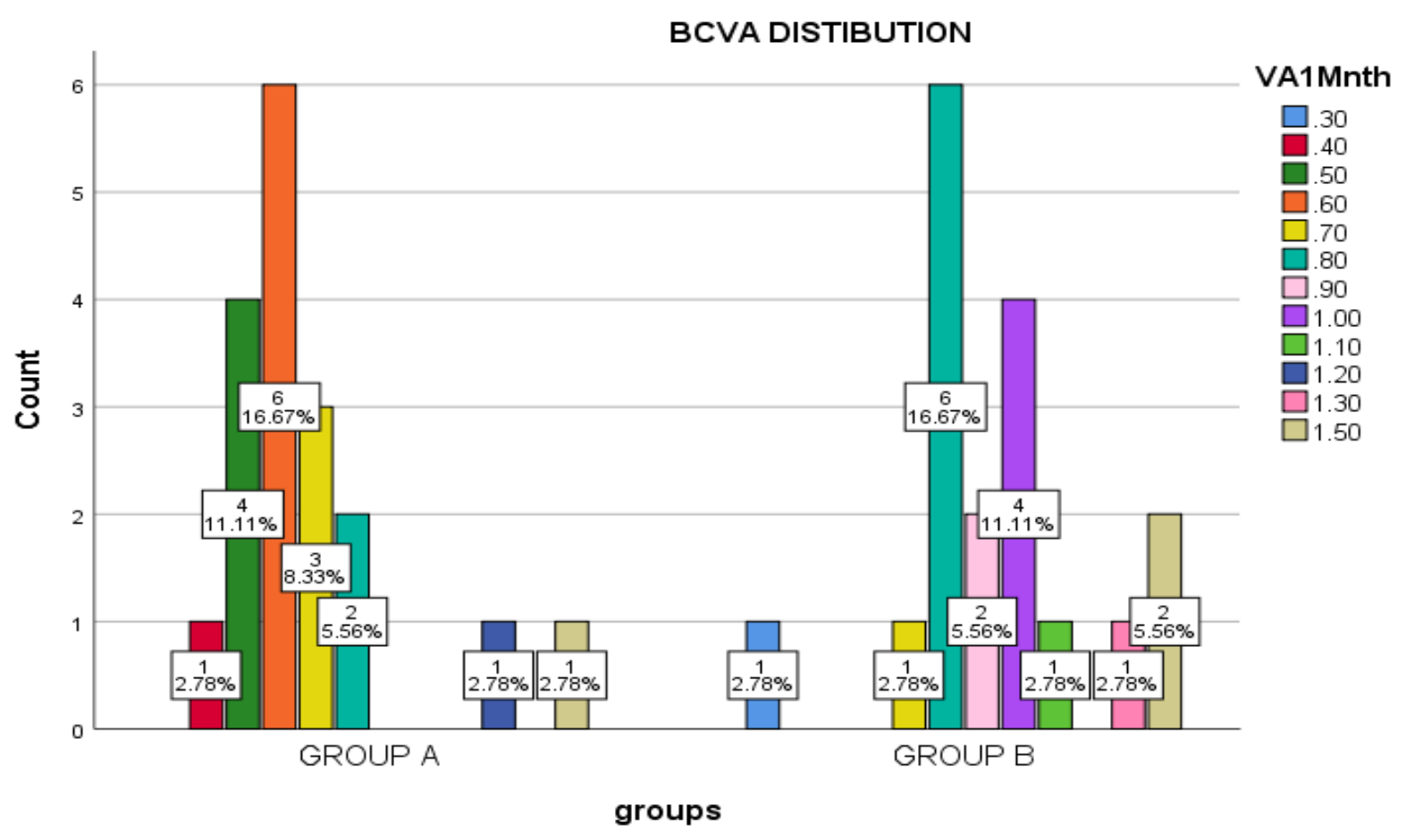

The BCVA at 1 month showed improvements in both groups, however the BCVA in group B was significantly lower than group A, P-value being < 0.05 .

The BCVA distribution at three months followup is shown in Figure 2.

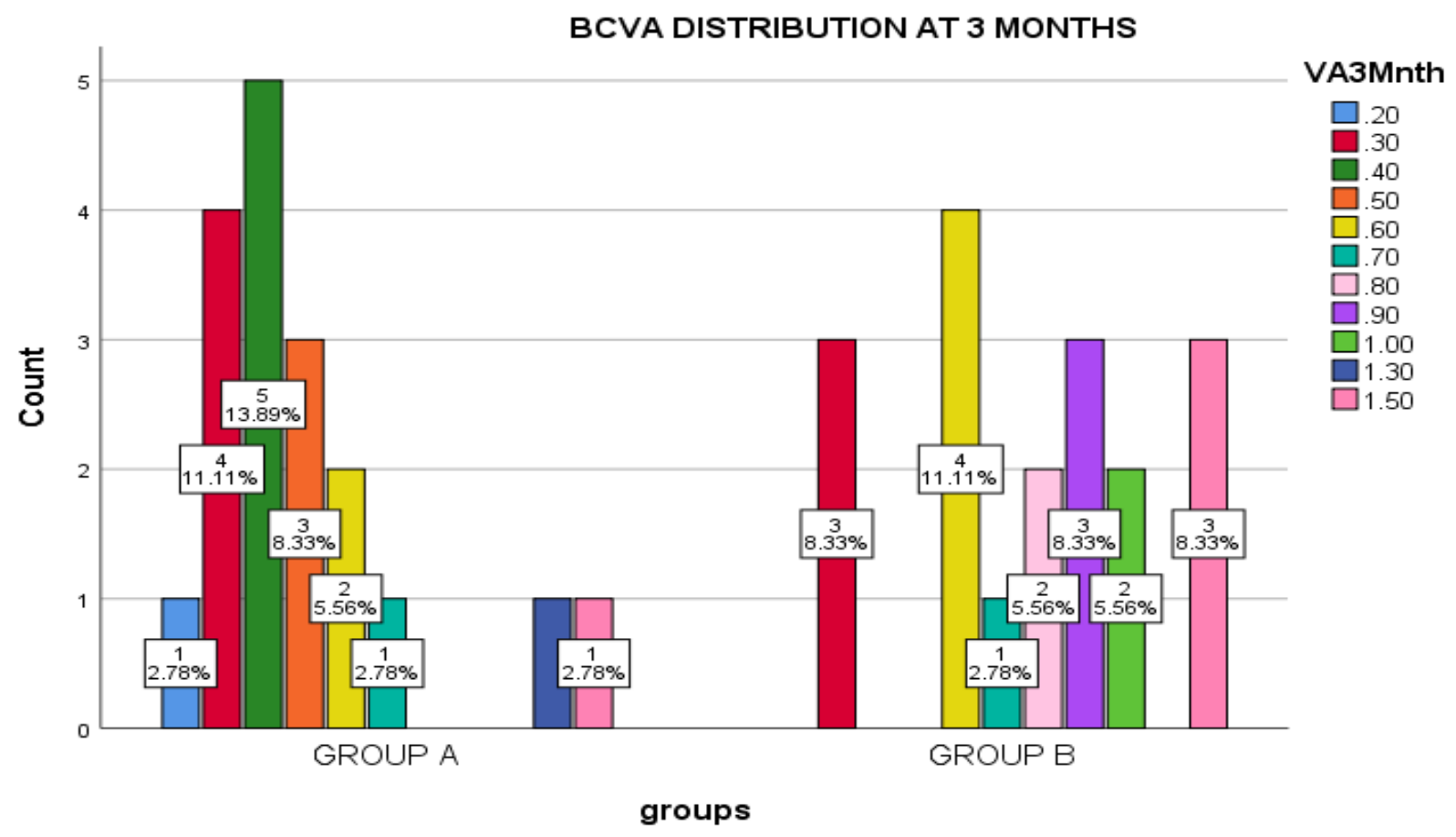

The BCVA at 3 month showed improvements in both groups, however the BCVA in group B was significantly lower than group A, P-value being $<0.05$.

Anatomical Outcome was seen as weather retina was attached or detached. The outcome is given in the following Figure 


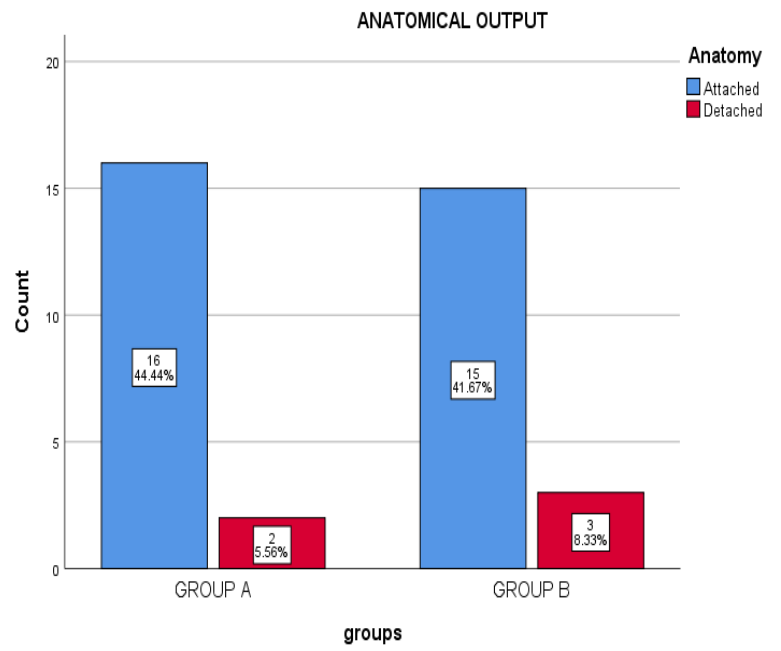

In Group A detachment was present in all follow-ups in 2/18 patients while in Group B detachment was present in all follow-ups in $3 / 18$ patients. The $\mathrm{P}$-value was $>0.05$ and thus the difference between two groups was insignificant.

\section{DISCUSSION}

In this study the anatomical and visual outcomes of rhegmatogenous retinal detachments were compared among two groups, Group A in which combined procedure in phacoemusification of clear lens was performed before the PPV and Group B in which PPV alone was performed. Visual outcome was recorded by Lomar chart at 3 intervals ( 1 week, 1 month and 3 months) and anatomical outcome was seen as whether retina was attached or detached.

It was seen that visual acuity did not differ among the two groups preoperatively and on first follow-up at 1 week. The $\mathrm{P}$ value being $>0.05$ in both. However, there was significant difference between the two group at 1 month and 3 months follow up, $\mathrm{P}$ value being $<0.05$ in each. The visual acuity was significantly better in Group A i.e combined phaco- vitrectomy.

For anatomical outcome no difference was seen between the groups. The $\mathrm{P}$ value was $>0.05$. So the visual outcome of group A was significantly better than group $\mathrm{B}$ while the anatomical outcome was the same.

There were number of complications which are mentioned percentage wise. In group A, $11 \%$ patients developed visually significant $\mathrm{PCO}$ and in group B, 44\% patients developed visually significant cataract. No Posterior capsule rupture occurred during phacoemulsification in both groups. On $1^{\text {st }}$ postoperative day, In Group A, 28\% patients had AC fibrin reaction However in Group B only $0.5 \%$ had AC fibrin reaction. In Group $11 \%$ patients had PS (Posterior
Synechea) at first follow-up on first week with one patient having inadequate dilatation. In Group B no such event happened. In Group B, 4 cases of accelerated cataract development were reported after lens touch by operating surgeon, and thus they needed early cataract surgery with IOL implantation. Both Groups reported corneal edema on $1^{\text {st }}$ post-opertive day in two patients. Both of these settled with topical medications. In immediate post-operative period, there was no other untoward complication.

In a study conducted by Young Gu et all, he compared the anatomical outcome and visual outcome after combined phaco vitrectomy and vitrectomy only. He observed that reattachment was seen in $84 \%$ patients after vitrectomy only vs $90 \%$ in patients who underwent combined phaco-vitrectomy procedure. However, it is important to note that the p-value was less than 0.05 , so that there was no statistical difference between the two groups on the basis of anatomical outcome. Also, as for visual acuity there was no statistically significant difference between the two group, $\mathrm{P}$ value being $>0.05$. However, in our study there was statistically significant difference between the visual acuities of two groups the combined phaco-vitrectomy with better visual outcome. ${ }^{10}$

Guber et al conducted a study with data of 1017 eyes with rhegmatogenous retinal detachment. 526 eyes underwent combined phaco-vitrectomy while remaining underwent vitrectomy only. He compared redetachment rates among two groups. A total of 103 eyes developed retinal re-detachment $(10.1 \%)$. It is important to note that there was no significant difference in the rate of re-detachment was found if additional phacoemulsification was performed. $\mathrm{P}$ value being > 0.05. ${ }^{11}$ However in our study no re-detachment occurred during follow-ups. These findings are consistent with my study which shows no difference in retinal attachment in both groups.

The trend is increasing towards combined procedure in single sitting as 23 , and 27 gauge vitrectomy is becoming the norm now along with further advances in the phacoemulsification techniques with small incisions. ${ }^{12}$ Combined phaco-vitrectomy with good success rate can be performed for a number of vitreous and retinal pathologies along with minimal complications such as rhegmatogenous $\mathrm{RD}$, advanced diabetic eye disease(ADED), full thickness macular holes (FTMH), vitreomacular interface disorders. ${ }^{13}$ However we performed the study on rhegmatogenous retinal detachments only with superior breaks only. Accelerated development of cataract after crystalline lens touch and also accelerated nuclear sclerosis is a common sequel after an un-eventful vitrectomy. ${ }^{14}$ it is 
also common finding that phacoemulsification is very challenging in patients with already vitrectomized eyes due to lack of vitreous support, unstable anterior chamber, very hard nucleus and possibility of PC rent with further complications. Also it is important to note that vitrectomy itself has gradually becoming a safe procedure because of small gauge vitrectomy, being sutureless and faster rehabilitation of the patients, and thus there gradual shift towards combined phacovitrectomy as it is being considered safe and effective procedure. ${ }^{14-15}$

\section{CONCLUSION}

This study concluded that combined phaco-vitrectomy has better visual outcome as compared to vitrectomy only while in terms of anatomical outcome none of these is superior to each other. It is important to note that the need of second surgery for cataract extraction is obviated in combined phaco vitrecomy as compared to vitrectomy only. There are multiple limitations to the study such as limited time duration, brief followup period and selection of only rhegmatogenous cases with exclusion of other retinal pathologies. Good points in this study include sinlge suregeon performing all surgeries and randomized control trials.

\section{REFERENCES}

1. Caiado RR, Magalhães O, Badaró E, Maia A, Novais EA, Stefanini FR, et al. Effect of lens status in the surgical success of 23-gauge primary vitrectomy for the management of rhegmatogenous retinal detachment: The Pan American Collaborative Retina Study (PACORES) group results. Retina. 2015;35(2):326-333.

2. Ajlan RS, Desai AA, Mainster MA. Endoscopic vitreoretinal surgery: principles, applications and new directions. Int J Retin Vitr. 2019;1-11.

3. Mompremier M, Adyanthaya R. Phacovitrectomy for the retina surgeon. Retin Today. 2014;(OCT 2014):56-58.

4. Sizmaz S, Esen E, Isik P, Cam B, Demircan N. Outcome and Complications of Combined Phacoemulsification and 23-Gauge Pars Plana Vitrectomy. J Ophthalmol. 2019;2019(November 2014):1-7.

5. Yes N, Yenerel NM. Comparison of intra- and postoperative complications of phaco between sequential and combined procedures of 23-gauge vitrectomy and phaco. 2017;
6. Al-Hinai AS. Vitrectomy versus Phaco-vitrectomy. Oman J Ophthalmol [Internet]. 2019;12(2):71-2. Available from: https://pubmed.ncbi.nlm.nih.gov/ 31198289

7. Williamson TOMH, Phth F. Incidence of lens touch during pars plana vitrectomy and outcomes from subsequent cataract surgery. 2016;825-829.

8. Tosi GM, Balestrazzi A, Baiocchi S, Tarantello A, Cevenini G, Marigliani D, et al. Complex retinal detachment in phakic patients: Previtrectomy phacoemulsification versus combined phacovitrectomy. Retina. 2017;37(4):630-636.

9. Karaca EE, Özdek Ş, Özmen MC, Dursun A, Yalçın NG. Safety of Polyacrylamide 1.5\% Left in Anterior Chamber in Combined Phacoemulsification and Pars Plana Vitrectomy Surgery. Curr Eye Res. 2016;41(4):501-506.

10. Gu BY, Sagong M, Chang WH. Phacovitrectomy versus Vitrectomy only for Primary Rhegmatogenous Retinal Detachment Repair. jkos [Internet]. 2011 May 31;52(5):537-543. Available from: http://dx.doi.org/10.3341/jkos.2011.52.5.537

11. Guber J, Bentivoglio M, Sturm V, Scholl HPN, Valmaggia C. Combined pars plana vitrectomy with phacoemulsification for rhegmatogenous retinal detachment repair. Clin Ophthalmol. 2019;13:15871591.

12. Lahey JM, Francis RR, Kearney JJ. Combining phacoemulsification with pars plana vitrectomy in patients with proliferative diabetic retinopathy: A series of 223 cases. Ophthalmology. 2003;

13. Demetriades AM, Gottsch JD, Thomsen R, Azab A, Stark WJ, Campochiaro PA, et al. Combined phacoemulsification, intraocular lens implantation, and vitrectomy for eyes with coexisting cataract and vitreoretinal pathology. Am J Ophthalmol. 2003;

14. Jalil A, Steeples L, Subramani S, Bindra MS, Dhawahir-Scala F, Patton N. Microincision cataract surgery combined with vitrectomy: A case series. Eye. 2014;

15. Dhawahir-Scala FE, Maino A, Saha K, Mokashi AA, McLauchlan R, Charles S. To posture or not to posture after macular hole surgery. Retina. 2008;

\section{AUTHOR'S CONTRIBUTIONS}

IM: Data Collection, Literature

ASK: Concept and Design, Critical Review

NS: Data analysis, Manuscript writing 